\title{
LUT
}

University

\section{Performance and Wake Comparison of Horizontal and Vertical Axis Wind Turbines under Varying Surface Roughness Conditions}

Mendoza Victor, Chaudhari Ashvinkumar, Goude Anders

This is a Final draft

version of a publication

published by Wiley

in Wind Energy

DOI: doi.org/10.1002/we.2299

Copyright of the original publication: (c) Wiley 2018

Please cite the publication as follows:

Mendoza, V., Chaudhari, A., Goude, A. (2018). Performance and Wake Comparison of Horizontal and Vertical Axis Wind Turbines under Varying Surface Roughness Conditions. Wind Energy. Pp. 1-15. DOI: doi.org/10.1002/we.2299

This is the peer reviewed version of the following article (cited above) which has been published in final form at https://doi.org/10.1002/we.2288. This article may be used for non-commercial purposes in accordance with Wiley Terms and Conditions for Use of Self-Archived Versions.

This is a parallel published version of an original publication. This version can differ from the original published article. 


\title{
Performance and Wake Comparison of Horizontal and Vertical Axis Wind Turbines under Varying Surface Roughness Conditions
}

\author{
VICTOR MENDOZA* \\ Department of Engineering Sciences, Division of Electricity, Uppsala University, Uppsala 751 21, Sweden \\ victor.mendoza@angstrom.uu.se \\ ASHVINKUMAR CHAUDHARI \\ CEID, School of Engineering Science, Lappeenranta University of Technology, \\ P.O. Box 20, 53851 Lappeenranta, Finland \\ ANDERS GOUDE \\ Department of Engineering Sciences, Division of Electricity, Uppsala University, Uppsala 751 21, Sweden
}

\begin{abstract}
A numerical study of both a horizontal axis wind turbine (HAWT) and a vertical axis wind turbine (VAWT) with similar size and power rating is presented. These large scale turbines have been tested when operating stand-alone at their optimal tip speed ratio (TSR) within a neutrally stratified ABL. The impact of three different surface roughness lengths on the turbine performance is studied for the both turbines. The turbines performance, the response to the variation in the surface roughness of terrain and the most relevant phenomena involved on the resulting wake were investigated. The main goal was to evaluate the differences and similarities of these two different types of turbine when they operate under the same atmospheric flow conditions. An actuator line model (ALM) was used together with the large eddy simulation (LES) approach for predicting wake effects, and it was implemented using the open-source CFD library Open-FOAM to solve the governing equations and to compute the resulting flow fields. This model was first validated using wind tunnel measurements of power coefficients and wake of interacting HAWTs, and then employed to study the wake structure of both full scale turbines. A preliminary study test comparing the forces on a VAWT blades against measurements was also investigated. These obtained results showed a better performance and shorter wake (faster recovery) for a HAWTcompared to a VAWT for the same atmospheric conditions.
\end{abstract}

Keywords: Atmospheric Boundary Layer (ABL), Vertical Axis Wind Turbines (VAWTs), Horizontal Axis Wind Turbines (HAWTs), Actuator Line Model (ALM), Dynamic Stall Model (DSM), Large Eddy Simulation (LES)

\footnotetext{
*Corresponding author
} 


\section{Introduction}

The majority of the currently deployed wind turbines are horizontal axis wind turbines (HAWTs). A renewed interest has been brought for vertical axis wine turbines (VAWTs) for offshore arrays, since they have several advantages over the conventional HAWTs, and their implementation can potentially mitigate the new challenges the offshore environment presents $[1,2,3]$. The omni-directionality allows them to operate with the incoming flow from any direction, further simplifying the mechanical design, since there is no need for a yawning mechanism (and often the pitching system). This characteristic is considerably appreciated in offshore environments where operation and maintenance are relevant items contributing in the total energy production cost. Another advantage of VAWTs is the availability to place the generator at the sea level, reducing the complexity involved in the installation and maintenance. This improves the stability of the overall structure and reduces the size and cost of the base, and moreover, it minimizes the concerns related to the dimensions and weight of the generator allowing the installation of heavy direct drive generators with permanent magnets [4]. On the other hand, VAWTs have a much lower power coefficients and suffer from vibration problems complicating their industrial models production. However, this study is limited to rather aerodynamic issues (power performance, wake development and recovery, etc.) and not to operational factors.

It is well known that the general structure of a turbine wake is directly related to the inflow characteristics, turbulence produced by the turbine, and operational conditions (blade pitch, yaw condition, tip speed ratio [TSR], etc). Considering both HAWT and VAWT stand-alone turbines, the common profile of velocity deficit is characterized by a Gaussian-like distribution (besides in the near wake) with a peak close to the region where the hub is located $[5,6]$. The near wake is where the main contribution of its structure comes directly from the turbine, while the far wake is characterized by (dissipative) turbulent structures, the recovery process, and a Gaussian profile of velocity. The turbulence intensity level in the incoming flow contributes considerably to a faster wake recovery as it has been reported in experimental $[7,8,9,10,11]$ and numerical studies [5, 12, 13, 14]. In a qualitative study [15] over the performance of a large wind farm, this effect showed that the downwind turbines decreased considerably the power deficit because of an increasing of the turbulence intensity within the incoming flow, highlighting the important role of the atmospheric turbulence in the total power output.

There are several well known studies in which the performance and wake characteristics of HAWTs [5, $16]$ and VAWTs $[6,17,18,19]$ were investigated; however, these works have been carried out separately for each type of turbine. Thus, a direct comparison in terms of aerodynamic performance between the two turbine types is perhaps difficult. The presented study provides results for both turbines under the same atmospheric flow conditions, with the aim of evaluating the differences and similarities of these two devices. Testing the both turbines with their best operating TSR conditions as well as under the same atmospheric flow conditions provided a fair comparison in terms of the aerodynamic performance, response to the atmospheric turbulence variation, and the resulting flow pattern. For this purpose, an actuator line model (ALM) has been implemented using the open-source computational fluid dynamics (CFD) toolbox library OpenFOAM $[20,21]$ to solve the governing equations and to compute the resulting wake.

Both an HAWT and a VAWT under a neutrally stratified atmospheric boundary layer (ABL) condition (i.e., no influence of vertical temperature profile) were tested for varying surface roughness conditions. These large scale turbines have similar size and power rating, operating at their optimal TSR. The HAWT employed is a well-documented large scale turbine NREL-5MW [22] and the VAWT is a proportionally scaled version of a 12-kW straight-bladed turbine $[23,24]$ with almost the same rotor projected area as the HAWT. 
The model is first validated using the wind tunnel measurements of the power coefficients and velocity flow field (wake) of two interacting HAWTs [25]. Additionally, the normal forces on one blade of a VAWT operating in an open site have been reproduced and compared with experimental data [23, 24]. The employed model is characterized by stability and accuracy, which makes it a potential tool in the design of large scale wind turbines.

\section{Methodology}

An ALM coupled to a dynamic stall model (DSM) has been employed to solve the blade force equations and model the turbine operations. The ALM samples the local velocity from the flow solver and then it calculates the angle of attack and relative velocity for each blade element, while the DSM calculates the unsteady lift and drag forces, which the ALM impart back as body forces into the flow solver. The present work is focused on evaluating the turbines aerodynamic performance and the wake modeling part. To do this, the library turbinesFoam developed by Bachant et al. [26, 27, 28] has been used as implementation of the ALM. To overcome the representation of a proper inlet boundary condition, the so-called recycling technique was used to generate the inflow turbulence for the flow. A detailed description of the ALM and DSM used in the present study can be found in [27] and [29], respectively, since only a brief explanation of the ALM is given further in this section.

\subsection{Actuator Line Model}

Based on the classical blade element theory, the ALM has been developed by Sørensen and Shen [30], and it is a three-dimensional and aerodynamic unsteady model used to study the resulting flow around (and within) wind turbines. This technique divides the blade into $n$-elements that have a two-dimensional airfoil behavior on which (normal and tangential) forces are determined using a DSM commonly based on empirical data.

The implementation of the ALM requires the values of the lift and drag coefficients for the different angles of attack and Reynolds numbers locally involved. The geometrical relation between the tangential speed of the blade $V_{\text {blade }}=\Omega_{r}$, where $\Omega$ is the angular velocity and $r$ is the radius to the element, and the incoming flow $V_{\text {in }}$ (which usually is smaller in magnitude than the free-stream velocity $V_{\infty}$ ) is used for the calculation of the relative flow $V_{\text {rel }}$ and the angle of attack $\alpha$,

$$
V_{\text {rel }}=V_{\text {in }}-V_{\text {blade }}
$$

The angle of relative wind $\varphi$ is represented by the sum of the angle of attack $\alpha$ and the blade pitch angle $\gamma$. Figure 1 shows an illustration of the velocities and acting forces on the cross-sectional airfoil element for both HAWTs and VAWTs. The inflow velocity considered in each element is the averaged velocity value of a number of samples around the element, which are symmetrically distributed. Once $\alpha$ and $V_{\text {rel }}$ are obtained (with the spanwise component removed from the latter), the lift and drag forces per spanwise length unit can be calculated as

$$
f_{L}=\frac{1}{2} \rho c C_{L}\left|V_{\text {rel }}\right|^{2}
$$




$$
f_{D}=\frac{1}{2} \rho c C_{D}\left|V_{\text {rel }}\right|^{2}
$$

where $C_{L}$ and $C_{D}$ are the lift and drag coefficients, respectively, which are dependent on $\alpha$ and the local Reynolds number. The lift component is perpendicular to $V_{\text {rel }}$ and the blade span component, while the drag has the same component as $V_{\text {rel }}$. The chord length is represented by $c$ and the density by $\rho$.

The same method is employed to obtain the forces on the towers, nacelle (for HAWTs) and struts (for VAWTs). Once all the forces in the lines of elements are calculated, these are added as a source of body force per unit of density into the momentum conservation equation 5 . The elements are moving in space, for every time-step within the fixed volume (domain), as they are in a turbine.

\subsection{The Large Eddy Simulation framework}

In order to use the LES approach the original Navier-Stokes equations have been filtered, and based on the incompressible flow case are expressed as

$$
\begin{aligned}
& \frac{\partial \tilde{u}_{i}}{\partial x_{i}}=0 \\
& \frac{\partial \tilde{u}_{i}}{\partial t}+\frac{\partial \tilde{u}_{i} \tilde{u}_{j}}{\partial x_{j}}=-\frac{1}{\rho} \frac{\partial \tilde{p}}{\partial x_{i}}+v \frac{\partial^{2} \tilde{u}_{i}}{\partial x_{j} \partial x_{j}}-\frac{f_{i}}{\rho}-\frac{\partial \tau_{i j}}{\partial x_{j}}
\end{aligned}
$$

with $\tilde{u}_{i}$ and $\tilde{p}$ representing the grid-filtered velocity and pressure values, respectively, $v$ the kinematic viscosity, $f_{i}$ the acting body forces (blades) and $\tau_{i j}$ the sub-grid scale (SGS) stress defined as $\tau_{i j}=$ $\widetilde{u_{i} u_{j}}-\tilde{u}_{i} \tilde{u}_{j}$.

In order to parameterize the deviatoric part of the SGS strees, the Smagorinsky model [31] was employed as

$$
\tau_{i j}-\frac{1}{3} \delta_{i j} \tau_{k k}=-2\left(C_{S} \widetilde{\Delta}\right)^{2}|\widetilde{S}|
$$

with $\widetilde{S}_{i j}=\frac{1}{2}\left(\frac{\partial \widetilde{u}_{i}}{\partial x_{j}}+\frac{\partial \widetilde{u}_{j}}{\partial x_{i}}\right)$ representing the resolved rate-of-strain tensor, $\widetilde{\Delta}$ is the grid size and $C_{S}=$ 0.1667 as the Smagorinsky constant (usually it has a value between 0.1 and 0.2 ).

\subsubsection{The recycling method for simulating the atmospheric boundary layer (ABL)}

In LES modelling, the correct reproduction of transient inflow condition is crucial for a proper modelling of ABL interaction to wind turbines. Previously, in many LES studies dealing with ABL modelling (e.g. $[32,33]$ ), a separate precursor LES calculation for ABL flow over a homogeneous terrain has been used to generate transient inflow boundary conditions. However, this method is very time consuming as it requires the entire simulation to be performed in two different stages: (1) precursor simulation for ABL flow over a horizontally-homogeneous flat surface (i.e. without turbine) to produce and store the instantaneous field data from each time-step, and (2) to utilize this time-dependent data for the main simulation (i.e., ABL with turbine).

In this study, the so-called recycling inflow method is employed for generating the fully developed ABL flow profiles before the turbines. Chaudhari et al. $[34,35,36]$ have studied the applicabilities of the recycling inflow method for $\mathrm{ABL}$ flow modelling over complex terrains, and further they have shown 
the validation of the method against field measurements. A more detailed description of the recycling method can be found in $[34,35,36]$, and only a brief explanation is given further in this section.

Using the recycling approach, the precursor simulation is combined with the main simulation, as shown in Figure 2. During the simulation, the flow variables, mainly velocity, temperature, SGS turbulent kinetic energy, etc., are sampled on a crosswind plane (i.e. recycling plane in Figure 2), which is sufficiently downstream from the inflow plane. The sampled data is then recycled back to the inflow plane. This process is repeated for each time step, creating a recycling section between the inflow and recycling planes, in which the flow becomes fully developed gradually. The method is very sensitive to the recycling length $L_{r}$ and the distance between the inflow and recycling planes. After testing various recycling lengths, Chaudhari et al. [34,36] suggested that the recycling length $L_{r}$ should be at least $3 \delta$ (i.e. $L_{r} \geq 3 \delta$ ), where $\delta$ is the ABL height and it is fixed to be $\delta=5 D$ (since the domain should be high enough to avoid any flow disturbances due to turbine wake [37, 38]). This restriction is needed to avoid any artificial turbulence structures within the recycling section due to too short recycling length. In addition to recycling the data, the method also uses the fixed velocity flux through the inflow plane (boundary) in order to maintain the same amount of volume flow rate throughout the entire simulation. The main advantage of the method is that precursor simulation is avoided, and the entire simulation is performed at once on a single computational domain as shown in Figure 2. The fully developed ABL profiles obtained using the recycling method are compared with the logarithmic profile and are presented in Figure 11 in Section 3.2.

\subsubsection{Wall-function modeling}

The surface boundary condition is also one of the challenges in LES modelling. In order to avoid massive computational resources required due to the finer mesh resolutions near the surface, the use of the wall-function approach has become standard in LES modeling of ABL flows (e.g. [16, 5, 34, 35, 36]). In addition, the surface roughness parameters (height or length) of a rough surface are often implemented via a wall-function model. In this work, a wall function model based on the well-known logarithmic law of a rough surface, implemented in OpenFOAM by Chaudhari et al. [34, 36], is used on the lower surface. The logarithmic law of the wall over rough surface is given by

$$
V_{x}=\frac{V_{x *}}{\kappa} \ln \left(\frac{z+z_{0}}{z_{0}}\right)
$$

where $z_{0}$ is the ground roughness length, $\kappa=0.41$ is the von-Kármán constant, and $V_{x *}$ is the instantaneous frictional velocity. More information on the implementation of this wall-function can be found in $[34,36,39]$.

\section{Results and Discussion}

In this section, results from the validation tests and the study of the influence of varying surface roughness conditions are presented. For the validation cases, similarities and discrepancies between numerical and experimental values are discussed. 


\subsection{Numerical model validation}

Two different experimental studies have been chosen to validate the employed numerical model. The first case is focused on the proper representation of the velocity field (wake structure) and the performance (power coefficients) of two interacting HAWTs within a wind tunnel, while the second one simulates the normal forces acting on the blades of a $12 \mathrm{~kW}$ VAWT, located in an open site.

\subsubsection{Two in-line wind turbines with spanwise offset}

The test case chosen for the power coefficient $\left(C_{P}\right)$ and wake validation is based on the experiment reported by Krogstad et al. in [25]. This work has been carried out in a wind tunnel facility and consists in two HAWTs, which are separated by a distance of $3 D$ in the streamwise direction and around $0.415 D$ $(0.4 \mathrm{~m})$ in the crosswind direction. The downstream rotor has a diameter of $D_{2}=0.894 \mathrm{~m}$ (further denoted as $D$ in this section) with a stepped tower consisting of four cylinders of different diameters while the upstream turbine has a slightly larger diameter of $D_{1}=0.944 \mathrm{~m}$ with and a tower with constant diameter. The arrangement of the experiment is such that the projection of the area from the upwind rotor covers half of the downwind turbine. The measurements of the streamwise velocity component were done in two spanwise lines, located $1 D$ and $3 D$ behind the downwind turbine at the height of the axis. Figures 3 and 4 show more details about the wind tunnel and the dimensions of the test configuration.

In this work, two different levels of turbulence have been tested. First, a turbulence intensity level of $T_{I}=0.23 \%$ in the location of the upwind turbine rotor, which corresponds to the measurements when the wind tunnel is empty and it is hereafter referred to as Case A. Then, in order to consider the effects of atmospheric turbulence, a large grid was used in the entrance of the chamber (shown in Figure 3) producing a higher level of turbulence intensity measured of $T_{I}=10 \%$, this is referred as Case B. The synthetic turbulence generator turbulentInlet, from the standard library of OpenFOAM, is employed to introduce the different levels of turbulence at the inlet of the studied domain. The ABL profile is not considered for the wind tunnel experiments, because the dimensions of the experimental chamber were not large enough to fully develop ABL conditions (see Figure 4). The employed lift and drag coefficients into the ALM are taken (digitized) from the work of Cakmakcioglu et al. [40], and they correspond to the Reynolds number equivalent to $R_{e}=10^{5}$. The upwind and downwind turbine are further denoted as $T_{1}$ and $T_{2}$, respectively. The domain has been discretized using a mesh topology with a uniform hexahedral distribution of cells in every direction, considering a grid resolution of $16.8 / D$ cells in the whole domain and a local refinement of $68 / D$ cells in the region around the rotor and behind the turbines in order to capture the details of the resulting wake. This mesh configuration has been chosen based on the criteria presented in [41] (as well for the meshes in subsequent sections). The DSM has not been used for these cases. The specifications of the tested turbines and experiments are listed in Table 1.

\section{Power and thrust coefficient curves}

Power coefficients of the both turbines are evaluated over a wide range of TSRs, but in the case of the downwind turbine, its performance has been obtained while the upwind turbine is operating at its optimal TSR of design $\lambda_{1}=\Omega_{1} R_{1} / V_{\infty}=6$, where $\Omega_{1}$ represents the angular speed of the upwind turbine rotor. The reference velocity considered for calculations is $V_{\infty}=10 \mathrm{~m} / \mathrm{s}$. The power and thrust 
coefficients of the turbines are defined as

$$
C_{P}=\frac{P}{\frac{1}{2} A \rho V_{\infty}^{3}}
$$

and

$$
C_{T}=\frac{T}{\frac{1}{2} A \rho V_{\infty}^{2}}
$$

with $P$ as the average power and $T$ as the average thrust for the rotor obtained over one revolution. Experimental and numerical results of the turbines performance for cases A and B are depicted in Figure 5 .

For the case A, with low turbulence levels, it is observed that there is a good agreement in the identification of the region where the turbines operate at the maximum power coefficient (optimal $\lambda$ ), and in general, with the trend of the curves. The numerical accuracy has to be highlighted for the power coefficient prediction in the upwind turbine for $\lambda_{1} \geq 5$, unlike for the lower TSRs, where discrepancies occur when reproducing the curve in the stall condition with a maximum error of $28 \%$. The numerical results of the downwind turbine show concordance with the lower TSRs, while there is an overestimation of the $C_{P}$ values for $\lambda_{2}>4$. The interaction of the wake with the downwind turbine is also captured and the power curve of $T_{2}$ is characterized by lower values than the curve of the upwind turbine due to the reduction in the available kinetic energy.

The experimental thrust coefficient data, which was expected to demonstrate increased $C_{T}$ values with increasing TSR, was very similar for both turbines, revealing that almost the same physical forces are applied on the rotors, while differing available kinetic energy. The upwind turbine has a considerably good agreement with the experimental values, while the numerical thrust coefficient curve of the downwind turbine shows an underestimation for all the tested TSRs, with a maximum error around of $12 \%$.

For the test case B, with high levels of turbulence on the freestream flow, again there is a good identification of the region where the turbines achieve the highest power coefficients. A better numerical representation of the power curve is made for $\lambda_{1}>4$ for the upwind turbine, and for the downwind turbine, this occurs at $\lambda_{2} \leq 5$, with a maximum error of $21 \%$. With respect to the thrust coefficients values, there is an overestimation in the upwind turbine at $\lambda_{2} \geq 4$ and an underestimation for the downwind one for all the studied TSRs with a maximum error around of $20 \%$.

\section{Wake}

The downwind turbine has been tested at three different TSRs, while the upwind turbine is operating at its optimal TSR $\lambda_{1}=6$, in order to study the resulting velocity field of interacting wakes. The three tested TSR conditions correspond to partially stall, optimal TSR and high TSR with $\lambda_{2}=3.5,4.75$ and 8.0, respectively. These conditions cover from the stall regime to the rotor almost working as a propeller.

As mentioned earlier, the streamwise velocity component has been measured in a horizontal line in the crosswind direction at the hub height at distances $x / D=1$ and $x / D=3$ behind the downwind turbine (see Figure 4), which allows us to identify the general structure of resulting flow from the interacting wakes. These results for the normalized streamwise velocity deficit profiles for all the tested cases and TSRs are displayed in Figure 6, where the radius $r$ has been used to normalize the spanwise position. 
In both cases, for the section $x / D=1$ behind the second rotor, the numerical and experimental results agree in the representation of the wake geometry and size, as well as the asymmetric behavior. Small discrepancies in some details of the velocity profile are revealed with a maximum error of $25 \%$ for particular regions. Three different regions can be identified in the velocity profile; in $-1.5<y / r<$ -0.5 where the resulting flow comes mainly from the upwind turbine, at $-0.5<y / r<0.5$ both turbines give a contribution to the wake, and $0.5<y / r<1.5$ where only the effects of the second rotor are present. A particular condition is observed for the highest TSR $\left(\lambda_{2}=8.0\right)$, since there is a relevant flow obstruction (velocity deficit) close to the blade tips due to the high rotational speed of the blades, and in the other hand, at the root location the opposite effect is noticed. Regarding the wake at $x / D=3$, a change is observed from an irregular shape of the velocity profile at $x / D=1$ to a smoothly one, specifically a Gaussian wake deficit profile. Therefore, in this section the wake recovery process already started and the direct contribution from the rotors into the flow is dissipated by the turbulent structures. In general, there is also a good agreement in numerical and experimental values in the regions outside the wake.

The effects of the added turbulence (Case B) are not significant in the near wake general structure (at $x / D=1$ ). Moreover at this location, it is noticed that for both cases A and B the velocity profiles do not differ considerably, more evident changes are present at the far wake section.

The authors believe that discrepancies between numerical and experimental values mainly can be caused by:

- The simplified implementation of the turbulence inlet generator is not realistic since it adds random noise to the specified inlet mean velocity from a defined turbulence level

- More detailed input data of $C_{L}$ and $C_{D}$ for a wider range of the Reynolds numbers is needed. Currently, only data for $R e=10^{5}$ has been considered which can not be appropriate for all the diversity of studied cases, since the employed ALM is highly sensitive to the input coefficients for a correct blade force projection

- Potential improvements of the numerical simulations can be achieved in the outer wake zones with the fully resolved wall boundary layer, which was not applied in this work

\subsubsection{ABL flow through a $12 \mathrm{~kW}$ straight-bladed VAWT in an open site}

A 3-bladed $12 \mathrm{~kW}$ VAWT located in the North of Uppsala (Sweden) has been chosen to validate the model under the influence of the ABL. The turbine has three rotor blades projected from a NACA0021 airfoil profile with a chord of $0.25 \mathrm{~m}$ and $6.48 \mathrm{~m}$ of diameter, the blade length is $5 \mathrm{~m}$. This turbine is placed at an open site and it is surrounded by mild vegetation which is mostly composed by grass and small bushes. The normal forces on one blade and its struts were measured using four load cells. The experimental activity and results as well as more detailed specifications of the device are available in [23] and [24]. These forces have been used as the validation parameters for a TSR of $\lambda=3.44$ (close to the optimal one). Additionally, obtained results for the same VAWT under the influence of a wind shear (only a constant mean wind profile without turbulence) are also shown for a comparison analysis. For this study, the operating conditions of the turbine are such that the freestream velocity at the blade equatorial plane $(z=5.75 \mathrm{~m})$ is $V_{\infty}=6.4 \mathrm{~m} / \mathrm{s}$. A roughness length of $z_{0}=0.025 \mathrm{~m}$ is considered for representing the place where the turbine is located. The lift and drag coefficients for the ALM are taken from the report of Sheldahl and Klimas [42]. The specifications of the modeled turbine are listed in Table 2. The employed discretization mesh has a hexahedral cell distribution over 
the whole domain with a resolution of $D / 3.24$ cells in the regions far from the turbine, considering several refinements levels up to $D / 26$ cells in the regions around and behind the rotor.

Figure 7 shows the instantaneous streamwise component of the velocity in the middle vertical plane for the turbine operating within the both conditions: ABL and a wind shear. For the ABL case, the flow turbulence is present over the whole studied region, while for the wind shear case it is only produced as an effect of the interaction between the main flow and the turbine. Since the upstream flow is naturally turbulent on the ABL, the resulting wake is shorter compared to the one produced under the wind shear influence (i.e. without turbulence).

\section{Normal force}

The normal force for one blade is defined as

$$
F_{N}=F_{L} \cos \varphi+F_{D} \sin \varphi
$$

where $F_{L}$ and $F_{C}$ are the lift and drag forces, respectively. The normal force response for one revolution is revealed in Figure 8 and numerical results under the influence of both the ABL and wind shear cases are compared against the experimental values. These results correspond to the averaged values for the last 5 turbine revolutions from a total of (at least) 40, in order to ensure the convergence of the simulations.

There is a good agreement, in terms of the amplitude (minimum and maximum values) and the trend of the force curve. In the ABL case, the force peak is overestimated at the upwind blade position close to $90^{\circ}$, and additionally, there is a considerable improvement in the representation of the force drop at $270^{\circ}$. The authors believe that this force drop arises from the turbulent flow effects produced by the ABL influence. A subsequent study for validating the normal forces at different TSRs was carried out, which shows that the model is not able to reproduce the force drop only considering a wind shear (without atmospheric turbulence).

\subsection{ABL flow through a full scale VAWT and a HAWT in open sites}

The ALM is employed to reproduce the ABL turbulent flow through two stand-alone turbines, a HAWT and a VAWT. In the simulations, the well documented NREL 5-MW turbine reported in [22] and a H-type VAWT are used, the latter turbine is a modified version (proportionally scaled) of the one studied in the subsection 3.1.2 in order to have a similar power rating as the NREL 5-MW turbine: the diameter of the HAWT is equal to the blade span length of the VAWT such that the merging of the both projected areas results in a circle within a square and their areas differ by a factor of $\pi / 4$. The VAWT rotor has a diameter $D=126 \mathrm{~m}$, with the same length as the blade span $H$. A standard NACA0021 airfoil profile with a chord $c$ of $5.25 \mathrm{~m}$ has been used for the cross-section of the blades, tapered a distance of $25 \mathrm{~m}$ from the tips until a chord of $3.15 \mathrm{~m}$ at the tips. The hub height $z_{h u b}$ (or equator height) of the turbine is $90 \mathrm{~m}$. An illustration of both tested turbines is shown in Figure 9.

Both simulated turbines have been tested in a wide range of TSRs in order to identify their optimal operating TSR for maximizing the $C_{P}$. The results are presented in Figure 10. The optimal TSRs are $\lambda=9.5$ and 3.5 for the HAWT and the VAWT, respectively.

These turbines were operated over different surface roughness conditions. However, in all three cases, the velocity at the hub height was fixed to $V_{\infty}=7.83 \mathrm{~m} / \mathrm{s}$, which is a reasonable speed for operating 
large scale turbines. Lift and drag coefficients of the blades are taken from the NREL technical report [22] for the HAWT, and from the XFOIL program [43] for the VAWT.

To generate the inflow boundary conditions, the recycling method for a fully developed ABL flow over a flat terrain has been employed. The considered domain has a dimension of $35 D \times 10 D \times 5 D$ in the $x, y$ and $z$ directions, respectively. The distance between the inflow plane and the recycling plane is $15 D$, and from the recycling plane to the turbine plane is $5 D$. A region with local refinement is located around and behind the turbines in order to capture the details close to the rotor and resulting wake. The refinement region covers a cross-section of $2 D \times 2 D$ (at ground level) and was extended in the streamwise direction till $16 D$ from the turbine, as it is depicted in Figure 2. The topology of the mesh has an hexaedral and uniform distribution of cells with a resolution of $31.5 / D$ cells, while the size is $63 / D$ cells at the locally refined region.

The inlet velocity profiles generated by the recycling method for all the cases are revealed in Figure 11. These numerical profiles have been compared against the results from the empirical equation of a logarithmic wind shear, and in both cases the reference velocity considered at the hub is $V_{\infty}=7.83 \mathrm{~m} / \mathrm{s}$. A good agreement between the numerical and empirical values is observed for all the cases.

Figure 12 displays the simulated instantaneous streamwise velocity at the vertical plane for both cases with different terrains. Important characteristics of the flow are represented as the wake location and shape, including the vertical wake shrinking (for VAWT) and the break location where the recovery process starts. Further, it have been observed that the increment in the roughness length leads to larger flow turbulence and hence a shorter the wake region. VAWT cases result in a larger flow blockage. For each surface roughness case, both turbines start from identical conditions, but their results differ due to the stochastical nature of the simulations.

Figures 13 and 14 show the normalized mean streamwise velocity on a vertical middle plane (at the center of the turbines) and in representative sections perpendicular to the flow, respectively, for all the surface roughness cases. In all cases, for the same type of turbine, the produced wakes are similar in shape and position. Additionally, it is possible to identify the location where the wake breaking starts the flow recovery process. In general, larger turbulence levels (i.e. higher surface roughness length) lead to faster wake recovery because of the improvement of the mixing process and momentum transfer (shorter wake extension in the streamwise direction). An asymmetric wake in the vicinity of the VAWT is clearly identified, which can be produced due to the large difference between the incoming flow velocity (in $x$-direction) and the blade's rotational speed going in the opposite direction (in $-x$-direction), since the velocity deficit is more pronounced in the positive $y$-direction. This asymmetry is amplified along the vertical profile by the wake interaction with the ground.

Table 3 reveals the aerodynamic performance of the turbines for the different surface conditions studied here. As expected, the HAWT achieves a higher $C_{P}$ for all the cases. However, the optimal performance of the HAWT is highly affected by the surface conditions showing that greater turbulence gives lower power, which is coherent with the previous results in [44, 45, 46, 47], in which $C_{P}$ decreases when the wind speeds are relatively close to the rated wind speed of the HAWT. In the case of the VAWT, a minor influence of the surface condition in the obtained $C_{P}$ is noticed. Similar results were obtained in the experimental work of Möllerström et al. [48], which showed a slightly higher efficiency of a VAWT at higher turbulence, proposing that the H-rotor is appropriate for wind sites with turbulent winds.

In order to do a qualitative comparison of the wake produced in the different studied cases, both horizontal and vertical profiles of normalized mean streamwise velocity components within the region of the wake are depicted in Figure 15. This comparison is made with respect to the logarithmic wind profile at the inlet of the domain for the different terrains. For the HAWT cases, the horizontal profile of 
the wake shows that it is almost axisymmetric with the turbine axis in the sections close to the rotor. Additionally at this location, this profile is characterized by an irregular shape which is dissipated to a smoothly Gaussian profile as the flow moves downstream in further sections due to the increasing mixing process. While the wake produced by the VAWT is concentrated in the central region of the rotor in both vertical and horizontal directions. A strong influence from the ground is observed in the vertical profiles. Differences between HAWT and VAWT velocity deficit are not relevant after the sections $x / D>7$.

\section{Conclusions}

The presented study evaluates the differences and similarities of both HAWT and VAWT stand-alone turbines when they operate at their optimal TSRs as well as under the same atmospheric flow conditions. This work, thus, provides a direct and fair comparison in terms of aerodynamic performance, sensitivity to atmospheric turbulence variation and the resulting wake of both turbine types. A neutrally-stratified ABL flow condition has been implemented, which is characterized by a logarithmic velocity profile, since there is no influence of vertical temperature profile (a key factor in the ABL configuration). The model was validated against measurements from different turbine types and operational conditions, showing good qualitative agreement in general.

There is a shorter resulting wake under the ABL condition (which is naturally turbulent) compared to the one produced under the influence of a wind shear (without atmospheric turbulence). In general, larger turbulence levels lead to faster wake recovery. This study gives a clear indication that it can not be expected to have a shorter wake for a VAWT compared to the one produced by a HAWT of similar power rating.

In general, a HAWT achieves higher power than a VAWT within the same flow conditions, however, the performance of the HAWT is much more sensitive to the surface conditions variations, decreasing the power production for higher turbulence level which is inherited by higher surface roughness.

The obtained results were coherent with conclusions of previous experimental works, separately for each type of turbine. However, to understand better the overall performance of each turbine type, further studies have to be carried out by varying relevant parameters, such as different aspect ratios for the VAWT rotors, complex terrains, non-neutral (stratified) ABL conditions, arrays, etc., since this work considers only two particular turbines which are operating stand-alone over a horizontally-homogeneous flat surface.

The same ALM has been employed considering different turbine types in a wide variety of studied cases, which is highly relevant since there is no need to modify the model or to employ a different one when performing the numerical studies. The relatively low computational cost, stability and accuracy of the employed model must be highlighted, which makes it a suitable for application in VAWTs simulations.

\section{Acknowledgments}

This work was conducted within the STandUP for Energy strategic research framework and is part of STandUP for Wind. The computational works were performed on resources provided by the Swedish National Infrastructure for Computing (SNIC) at NSC. 


\section{References}

[1] M. Borg, M. Collu, and F. Brennan, "Offshore floating vertical axis wind turbines: advantages, disadvantages, and dynamics modelling state of the art," in The International Conference on Marine \& Offshore Renewable Energy (MORE 2012), pp. 26-27, RINA HQ London, 2012.

[2] S. Peace, "Another approach to wind: vertical-axis turbines may avoid the limitations of today's standard propeller-like machines," Mechanical Engineering-CIME, vol. 126, no. 6, pp. 28-32, 2004.

[3] P. Musgrove, "Wind energy conversion: recent progress and future prospects," Solar \& wind technology, vol. 4, no. 1, pp. 37-49, 1987.

[4] S. Eriksson, A. Solum, M. Leijon, and H. Bernhoff, "Simulations and experiments on a $12 \mathrm{~kW}$ direct driven pm synchronous generator for wind power," Renewable Energy, vol. 33, no. 4, pp. 674-681, 2008.

[5] Y.-T. Wu and F. Porté-Agel, "Atmospheric turbulence effects on wind-turbine wakes: An les study," Energies, vol. 5, no. 12, pp. 5340-5362, 2012.

[6] M. Abkar and J. O. Dabiri, "Self-similarity and flow characteristics of vertical-axis wind turbine wakes: an LES study," Journal of Turbulence, vol. 18, no. 4, pp. 373-389, 2017.

[7] L. P. Chamorro and F. Porté-Agel, "A wind-tunnel investigation of wind-turbine wakes: boundarylayer turbulence effects," Boundary-layer meteorology, vol. 132, no. 1, pp. 129-149, 2009.

[8] D. Medici and P. Alfredsson, "Measurements on a wind turbine wake: $3 \mathrm{~d}$ effects and bluff body vortex shedding," Wind Energy, vol. 9, no. 3, pp. 219-236, 2006.

[9] T. Maeda, Y. Kamada, J. Murata, S. Yonekura, T. Ito, A. Okawa, and T. Kogaki, "Wind tunnel study on wind and turbulence intensity profiles in wind turbine wake," Journal of Thermal Science, vol. 20, no. 2, pp. 127-132, 2011.

[10] W. Zhang, C. D. Markfort, and F. Porté-Agel, "Near-wake flow structure downwind of a wind turbine in a turbulent boundary layer," Experiments in fluids, vol. 52, no. 5, pp. 1219-1235, 2012.

[11] Q. Li, T. Maeda, Y. Kamada, T. Ogasawara, A. Nakai, and T. Kasuya, "Investigation of power performance and wake on a straight-bladed vertical axis wind turbine with field experiments," Energy, vol. 141, pp. 1113-1123, 2017.

[12] N. Troldborg, J. N. Sørensen, and R. Mikkelsen, "Actuator line simulation of wake of wind turbine operating in turbulent inflow," Journal of physics: conference series, vol. 75, no. 1, p. 012063 , 2007.

[13] P.-E. Réthoré, N. N. Sørensen, A. Bechmann, and F. Zhale, "Study of the atmospheric wake turbulence of a cfd actuator disc model," in 2009 European Wind Energy Conference and Exhibition, (Marseille, France), pp. 1-9, 2009.

[14] V. Mendoza and A. Goude, "Wake flow simulation of a vertical axis wind turbine under the influence of wind shear," Journal of Physics: Conference Series, vol. 854, no. 1, p. 012031, 2017.

[15] K. S. Hansen, R. J. Barthelmie, L. E. Jensen, and A. Sommer, "The impact of turbulence intensity and atmospheric stability on power deficits due to wind turbine wakes at horns rev wind farm," Wind Energy, vol. 15, no. 1, pp. 183-196, 2012. 
[16] F. Porté-Agel, Y.-T. Wu, H. Lu, and R. J. Conzemius, "Large-eddy simulation of atmospheric boundary layer flow through wind turbines and wind farms," Journal of Wind Engineering and Industrial Aerodynamics, vol. 99, no. 4, pp. 154-168, 2011.

[17] S. Shamsoddin and F. Porté-Agel, "A large-eddy simulation study of vertical axis wind turbine wakes in the atmospheric boundary layer," Energies, vol. 9, no. 5, 2016.

[18] V. F.-C. Rolin and F. Porté-Agel, "Experimental investigation of vertical-axis wind-turbine wakes in boundary layer flow," Renewable Energy Bulletin A, vol. 118, pp. 1-13, 2018.

[19] S. H. Hezaveh, E. Bou-Zeid, M. W. Lohry, and L. Martinelli, "Simulation and wake analysis of a single vertical axis wind turbine," Wind Energy, vol. 20, no. 4, pp. 713-730, 2017.

[20] H. G. Weller, G. Tabor, H. Jasak, and C. Fureby, "A tensorial approach to computational continuum mechanics using object-oriented techniques," Computers in Physics, vol. 12, no. 6, pp. 620-631, 1998.

[21] OpenFOAM, OpenFOAM User Guide. 2017. The OpenFOAM Foundation Ltd., PO Box 56676 London, UK.

[22] J. Jonkman, S. Butterfield, W. Musial, and G. Scott, "Definition of a 5-MW reference wind turbine for offshore system development," tech. rep., National Renewable Energy Lab.(NREL), Golden, CO (United States), 2009.

[23] M. Rossander, E. Dyachuk, S. Apelfröjd, K. Trolin, A. Goude, H. Bernhoff, and S. Eriksson, "Evaluation of a blade force measurement system for a vertical axis wind turbine using load cells," Energies, vol. 8, no. 6, pp. 5973-5996, 2015.

[24] E. Dyachuk, M. Rossander, A. Goude, and H. Bernhoff, "Measurements of the aerodynamic normal forces on a 12-kw straight-bladed vertical axis wind turbine," Energies, vol. 8, no. 8, pp. 8482-8496, 2015.

[25] P.-Å. Krogstad, L. Sætran, and M. S. Adaramola, "Blind test 3 calculations of the performance and wake development behind two in-line and offset model wind turbines," Journal of Fluids and Structures, vol. 52, pp. 65-80, 2015.

[26] P. Bachant and M. Wosnik, "Simulating wind and marine hydrokinetic turbines with actuator lines in rans and les," in APS Meeting Abstracts, (Boston, Massachusetts-USA), 2015.

[27] P. Bachant, A. Goude, and M. Wosnik, "Actuator line modeling of vertical-axis turbines," arXiv preprint arXiv:1605.01449, 2016.

[28] P. Bachant, A. Goude, and M. Wosnik, “turbinesfoam: v0.0.7,” Apr. 2016.

[29] E. Dyachuk, Aerodynamics of vertical axis wind turbines: Development of simulation tools and experiments. PhD thesis, Acta Universitatis Upsaliensis, 2015.

[30] J. N. Sørensen and W. Z. Shen, "Computation of wind turbine wakes using combined navierstokes/actuator-line methodology," in 1999 European Wind Energy Conference and Exhibition, (Nice-France), pp. 156-159, 1999.

[31] J. Smagorinsky, "General circulation experiments with the primitive equations: I. the basic experiment," Monthly weather review, vol. 91, no. 3, pp. 99-164, 1963. 
[32] A. Silva Lopes, J. Palma, and F. Castro, "Simulation of the Askervein flow. Part 2: Large-eddy simulations," Boundary-Layer Meteorol., vol. 125, no. 1, pp. 85-108, 2007.

[33] M. Diebold, C. Higgins, J. Fang, A. Bechmann, and M. Parlange, "Flow over hills: A Large-Eddy Simulation of the Bolund case," Boundary-Layer Meteorol., vol. 148, no. 1, pp. 177-194, 2013.

[34] A. Chaudhari, Large-eddy simulation of wind flows over complex terrains for wind energy applications. PhD thesis, Lappeenranta University of Technology, 2014.

[35] A. Chaudhari, A. Hellsten, and J. Hämäläinen, "Full-scale experimental validation of large-eddy simulation of wind flows over complex terrain: The bolund hill," Advances in Meteorology, vol. 2016, p. 14 pages, 2016. Article ID 9232759.

[36] A. Chaudhari, V. Vuorinen, J. Hämäläinen, and A. Hellsten, "Large-eddy simulations for hill terrains: validation with wind-tunnel and field measurements," Computational and Applied Mathematics, vol. 37, pp. 2017-2038, 2018.

[37] S. Xie and C. Archer, "A numerical study of wind-turbine wakes for three atmospheric stability conditions," Boundary-Layer Meteorol, vol. 165, pp. 87-112, 2017.

[38] A. Chaudhari, O. Agafonova, A. Hellsten, and J. Sorvari, "Numerical study of the impact of atmospheric stratification on a wind-turbine performance," Journal of Physics: Conference Series, vol. 854, no. 1, p. 012007, 2017.

[39] V. Vuorinen, A. Chaudhari, and J.-P. Keskinen, "Large-eddy simulation in a complex hill terrain enabled by a compact fractional step openfoam solver," Advances in Engineering Software, vol. 79, no. 0 , pp. $70-80,2015$.

[40] S. Cakmakcioglu, I. Sert, O. Tugluk, and N. Sezer-Uzol, "2-d and 3-d cfd investigation of nrel s826 airfoil at low reynolds numbers," vol. 524, no. 1, p. 012028, 2014.

[41] P. Jha, M. Churchfield, P. Moriarty, and S. S., "Guidelines for volume force distributions within actuator line modeling of wind turbines on large-eddy simulation-type grids," ASME. J. Sol. Energy Eng., vol. 136, no. 3, pp. 031003-031003-11, 2014.

[42] R. E. Sheldahl and P. C. Klimas, "Aerodynamic characteristics of seven symmetrical airfoil sections through 180-degree angle of attack for use in aerodynamic analysis of vertical axis wind turbines," tech. rep., Sandia National Labs., Albuquerque, NM (USA), 1981.

[43] M. Drela, "XFOIL: An analysis and design system for low reynolds number airfoils," in Low Reynolds number aerodynamics, vol. 54, pp. 1-12, New York, Springer, 1989.

[44] A. Honrubia, A. Vigueras-Rodríguez, and E. Gómez-Lázaro, "The influence of turbulence and vertical wind profile in wind turbine power curve," in Progress in turbulence and wind energy IV, pp. 251-254, Springer, 2012.

[45] K. Kaiser, W. Langreder, H. Hohlen, and J. Højstrup, "Turbulence correction for power curves," in Wind Energy (J. Peinke, P. Schaumann, and S. Barth, eds.), (Berlin, Heidelberg), pp. 159-162, Springer Berlin Heidelberg, 2007.

[46] R. Wagner, M. Courtney, T. Larsen, and U. Schmidt Paulsen, Simulation of shear and turbulence impact on wind turbine performance. Danmarks Tekniske Universitet, Risø Nationallaboratoriet for Bæredygtig Energi, 2010. 
[47] J. Gottschall and J. Peinke, "How to improve the estimation of power curves for wind turbines," Environmental Research Letters, vol. 3, no. 1, p. 015005, 2008.

[48] E. Möllerström, F. Ottermo, A. Goude, S. Eriksson, J. Hylander, and H. Bernhoff, "Turbulence influence on wind energy extraction for a medium size vertical axis wind turbine," Wind Energy, vol. 19, no. 11, pp. 1963-1973, 2016.

[49] P. Krogstad and L. Sætran, "Invitation to the 2013 blind test 3 workshop two in-line wind turbines with spanwise offset," Department of Energy and Process Engineering, NTNU, Trondheim, Norway, 2013. 
Table 1: Nominal parameters of the tested turbines and experiments.

\begin{tabular}{|c|c|c|c|c|c|c|c|}
\hline & \multicolumn{3}{|c|}{ Turbines } & \multicolumn{4}{|c|}{ Experimental cases } \\
\hline & $T_{1}$ & & $T_{2}$ & & A & & $\mathrm{B}$ \\
\hline Number of blades & & 3 & & $V_{\infty}[\mathrm{m} / \mathrm{s}]$ & & 10 & \\
\hline Diameter [m] & 0.944 & & 0.894 & $T_{I}[\%]$ & 0.23 & & 10 \\
\hline Hub height [m] & & 0.817 & & & & & \\
\hline Blade profile & & NREL S826 & & & & & \\
\hline Chord length [m] & & variable & & & & & \\
\hline
\end{tabular}

Table 2: Nominal parameters of the modeled 12kW VAWT.

\begin{tabular}{ll}
\hline \hline Number of blades & 3 \\
Diameter $[\mathrm{m}]$ & 6.48 \\
Hub height $[\mathrm{m}]$ & 6 \\
Blade profile & NACA0021 \\
Chord length $[\mathrm{m}]$ & 0.25 \\
$V_{\infty}[\mathrm{m} / \mathrm{s}]$ & 6.4 \\
TSR & 3.44 \\
\hline \hline
\end{tabular}

Table 3: Aerodynamic performance of the tested turbines for the different terrains.

\begin{tabular}{cccccc}
\hline Turbine & $z_{0}[\mathrm{~m}]$ & $C_{P}$ & $P[\mathrm{MW}]$ & $C_{T}$ & $T[\mathrm{MW}]$ \\
\hline \multirow{3}{*}{ VAWT } & 0.0005 & 0.346 & 1.579 & 0.688 & 0.402 \\
& 0.025 & 0.321 & 1.500 & 0.702 & 0.410 \\
& 0.1 & 0.338 & 1.546 & 0.673 & 0.393 \\
\hline \multirow{2}{*}{ HAWT } & 0.0005 & 0.558 & 2.003 & 0.870 & 0.399 \\
& 0.025 & 0.556 & 1.997 & 0.860 & 0.394 \\
& 0.1 & 0.496 & 1.780 & 0.761 & 0.348 \\
\hline
\end{tabular}



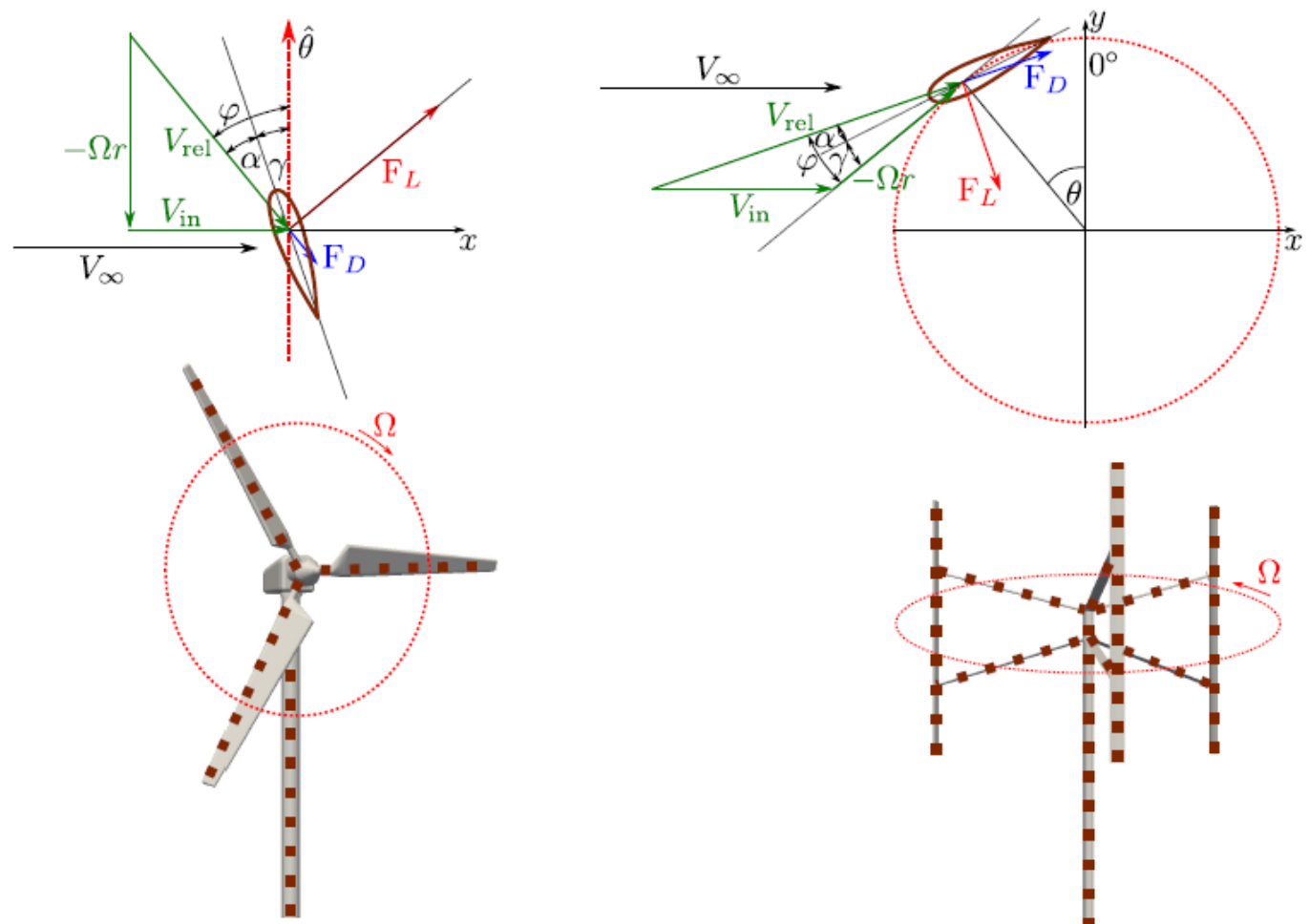

Figure 1: Illustration of velocity vectors and forces acting at the cross-section airfoil element for a HAWT (left) and a VAWT (right) and a schematic with the lines of elements. Note: For HAWTs, $\hat{\theta}$ denotes the tangential direction of the blade while $\theta$ is the azimuthal angle for VAWTs

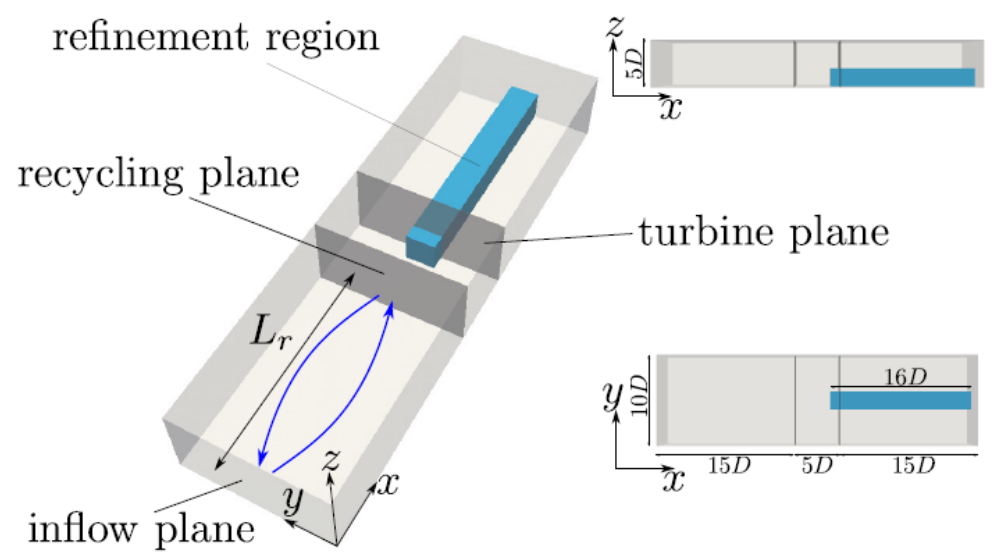

Figure 2: Schematic views of the domain and relevant dimensions for the application of the recycling method: perspective (left), side (upper right) and top (lower right). 

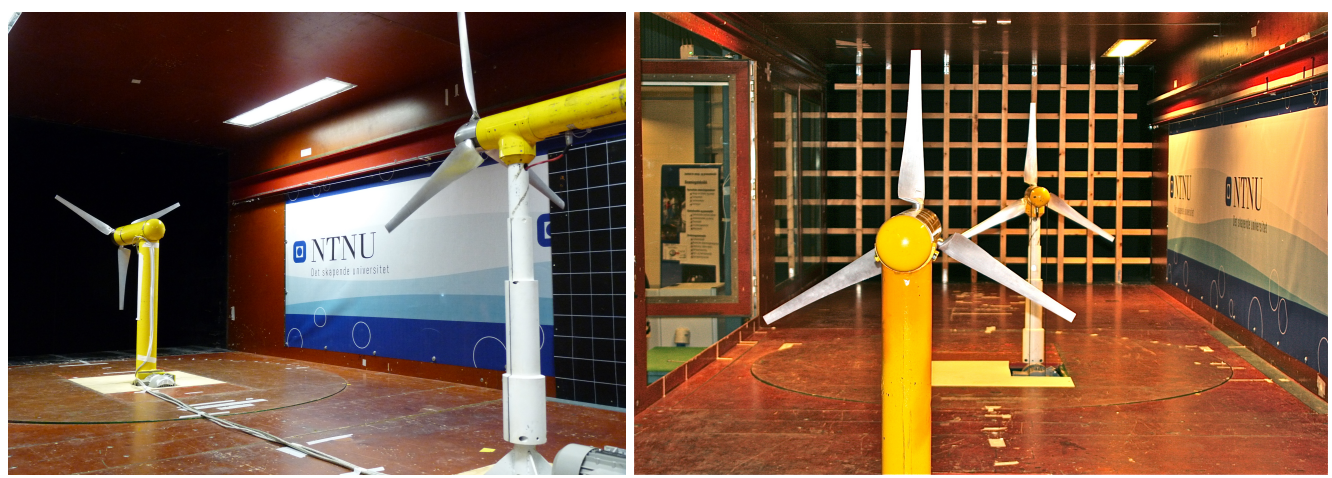

Figure 3: Model in the wind tunnel [49]: perspective (left) and from downwind (right) views.
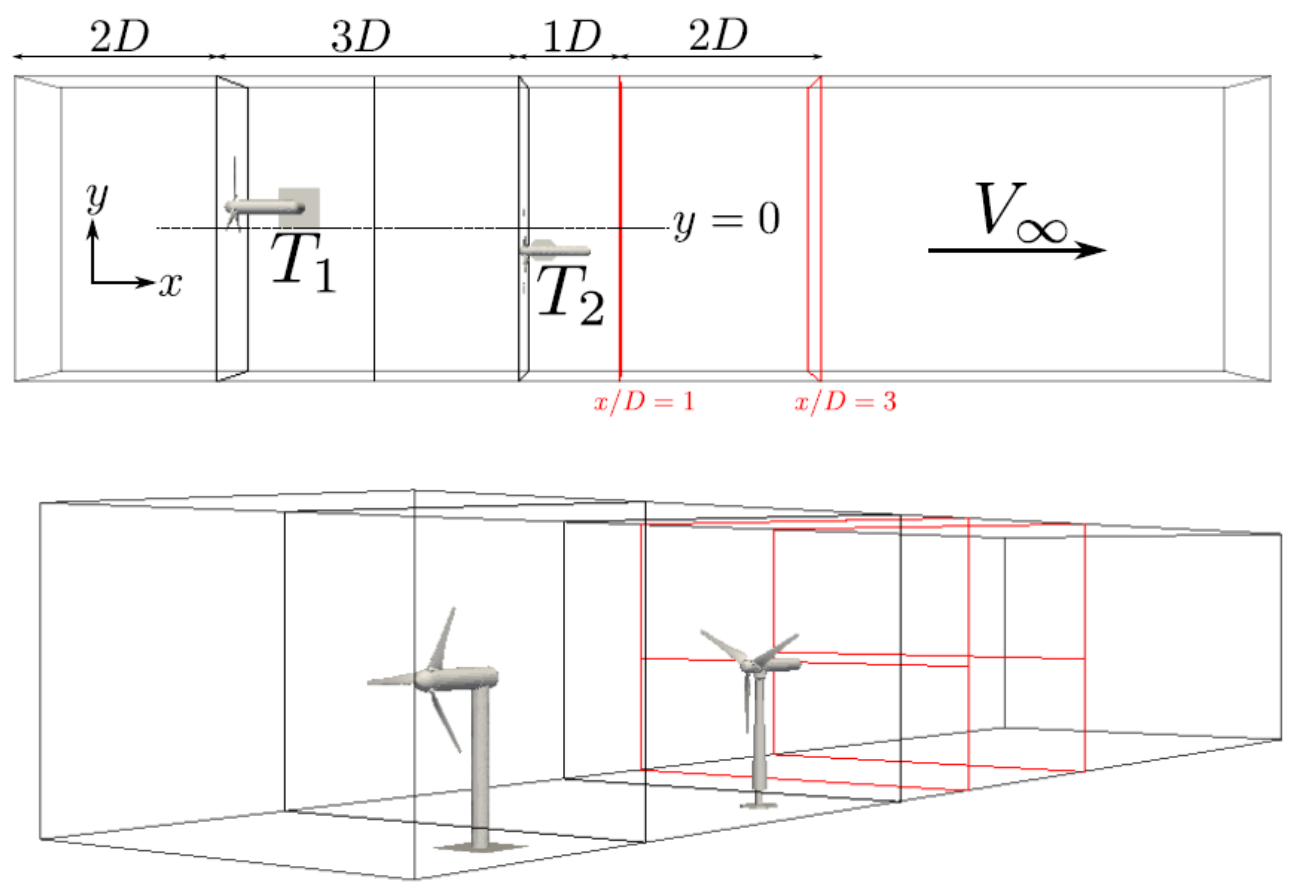

Figure 4: Schematic view of the wind tunnel domain: from the upper part (top) and perspective (bottom). The first two perpendicular sections (in black) represent the rotor planes of the turbines, while the two sections after the turbines (in red) represent the plane where the measurements were done, specifically in a crosswind line at the rotor height. 

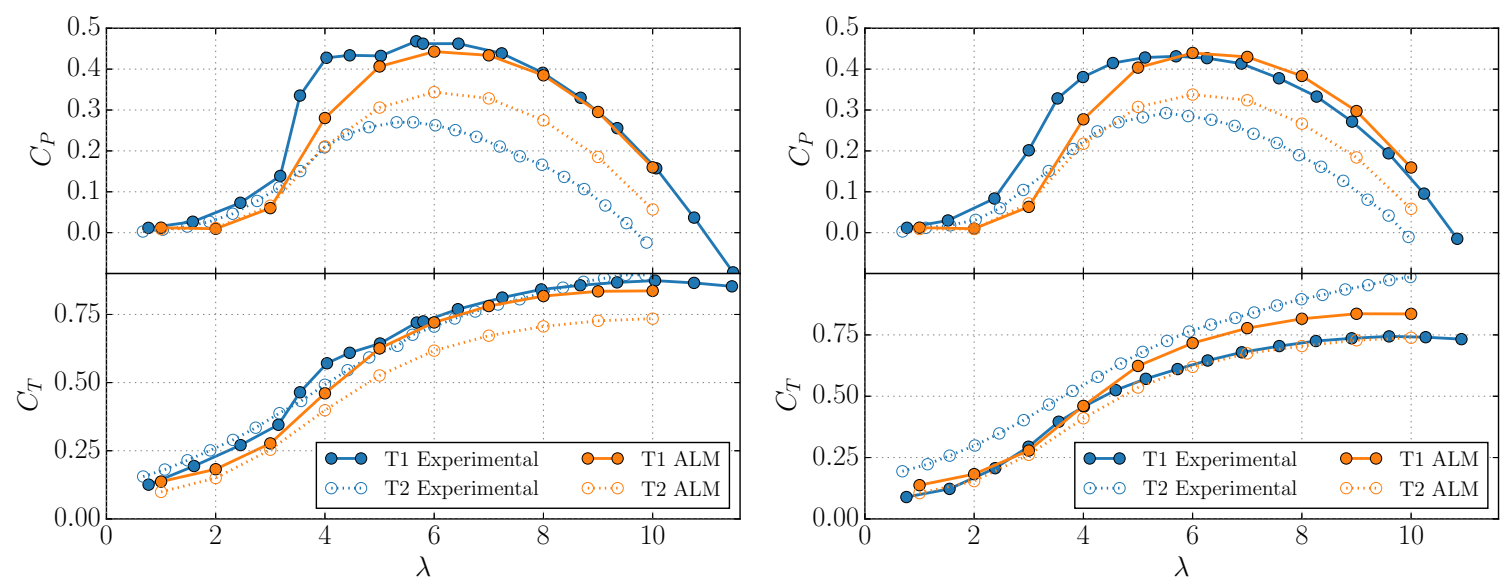

Figure 5: Power coefficient and thrust coefficient for the case A with low turbulence level (left) and the case B with high turbulence level (right).
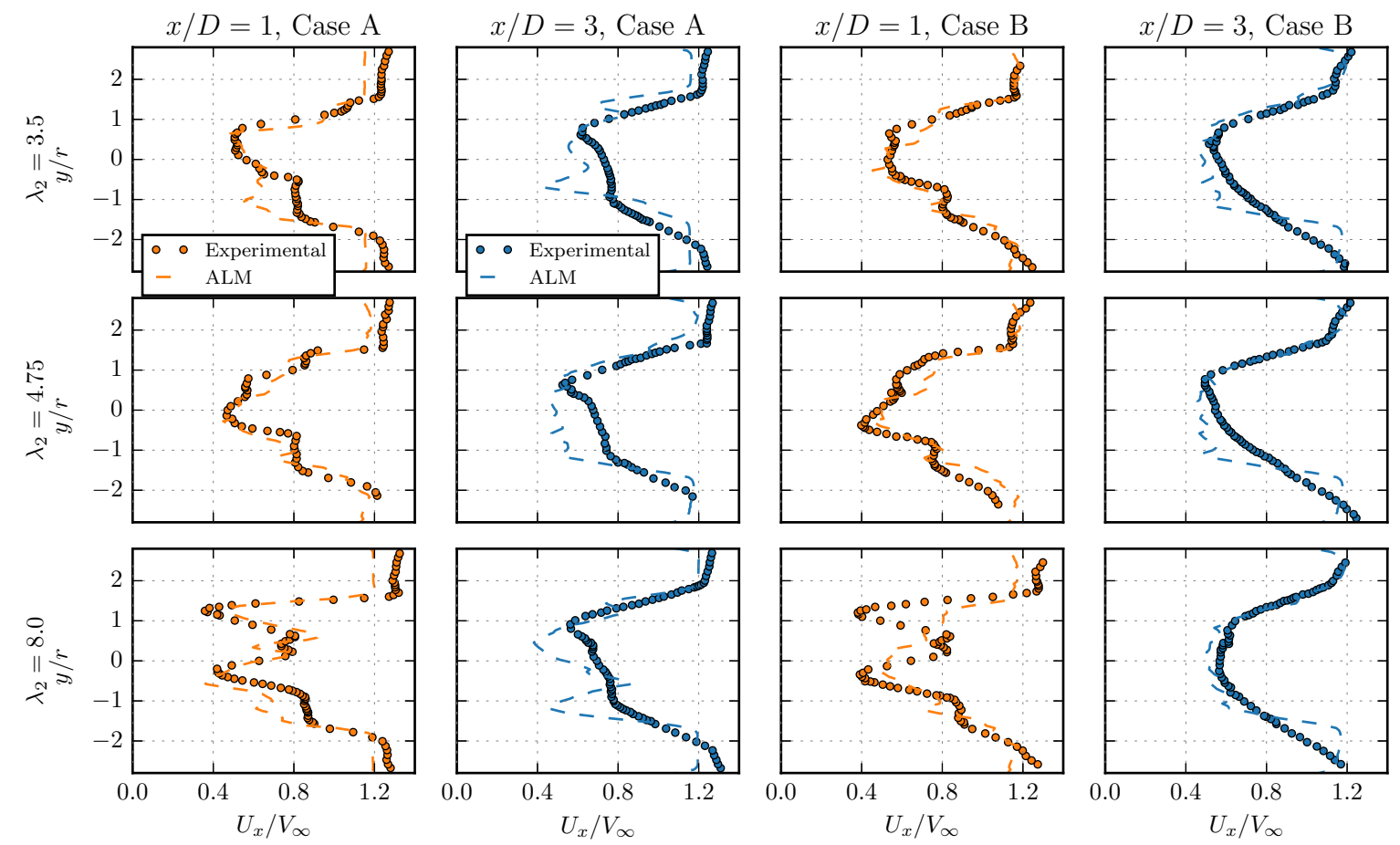

Figure 6: Normalized mean streamwise velocity profiles along a crosswind (horizontal) line through the rotor center. 


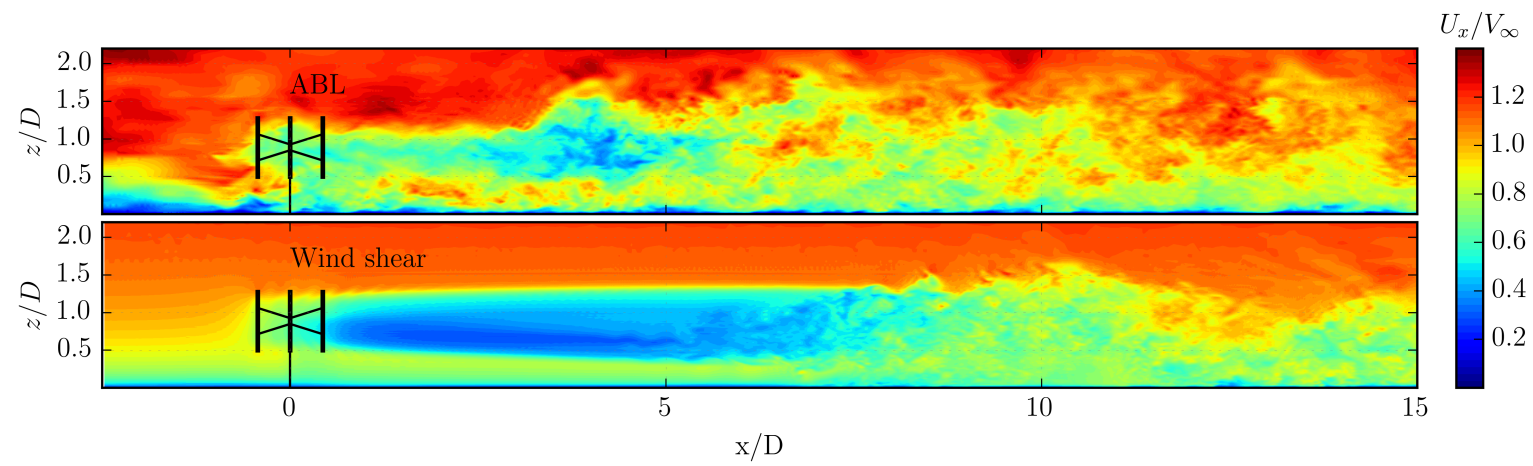

Figure 7: Normalized instantaneous streamwise velocity at the vertical middle plane: ABL (top) and wind shear (bottom).

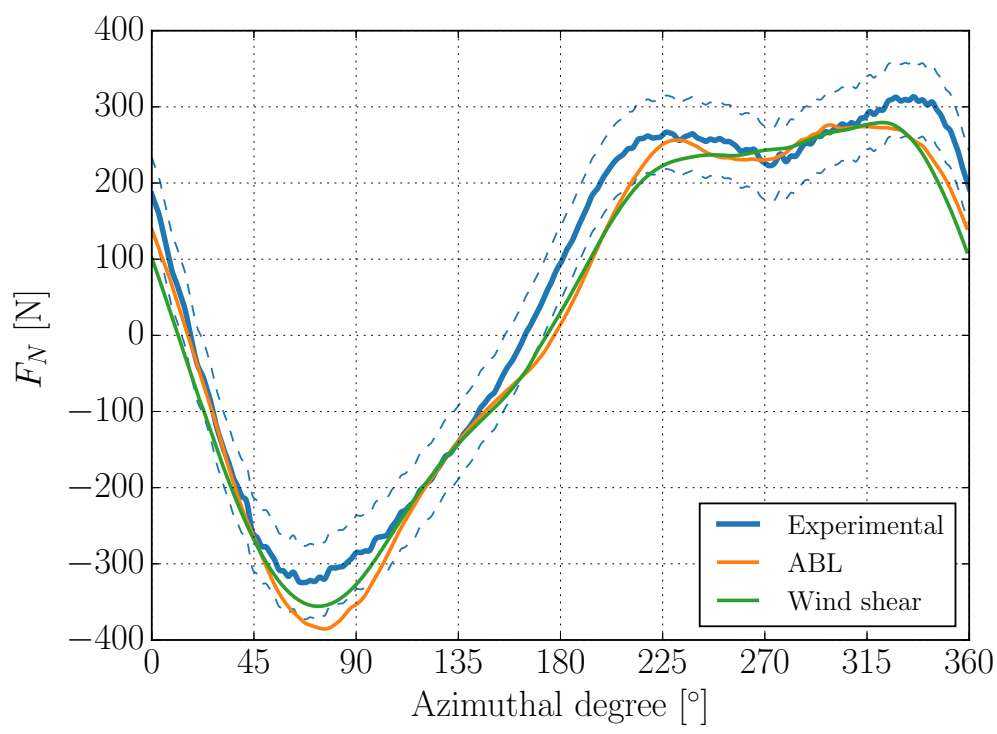

Figure 8: The normal force response under the influence of the ABL and a wind shear.

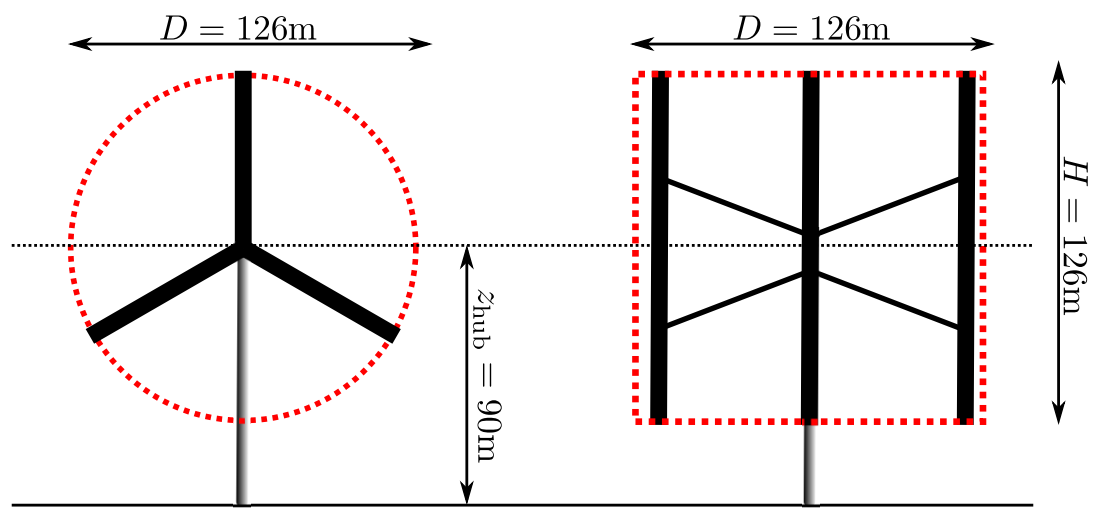

Figure 9: Illustration of the main characteristic dimensions of the tested turbines. 


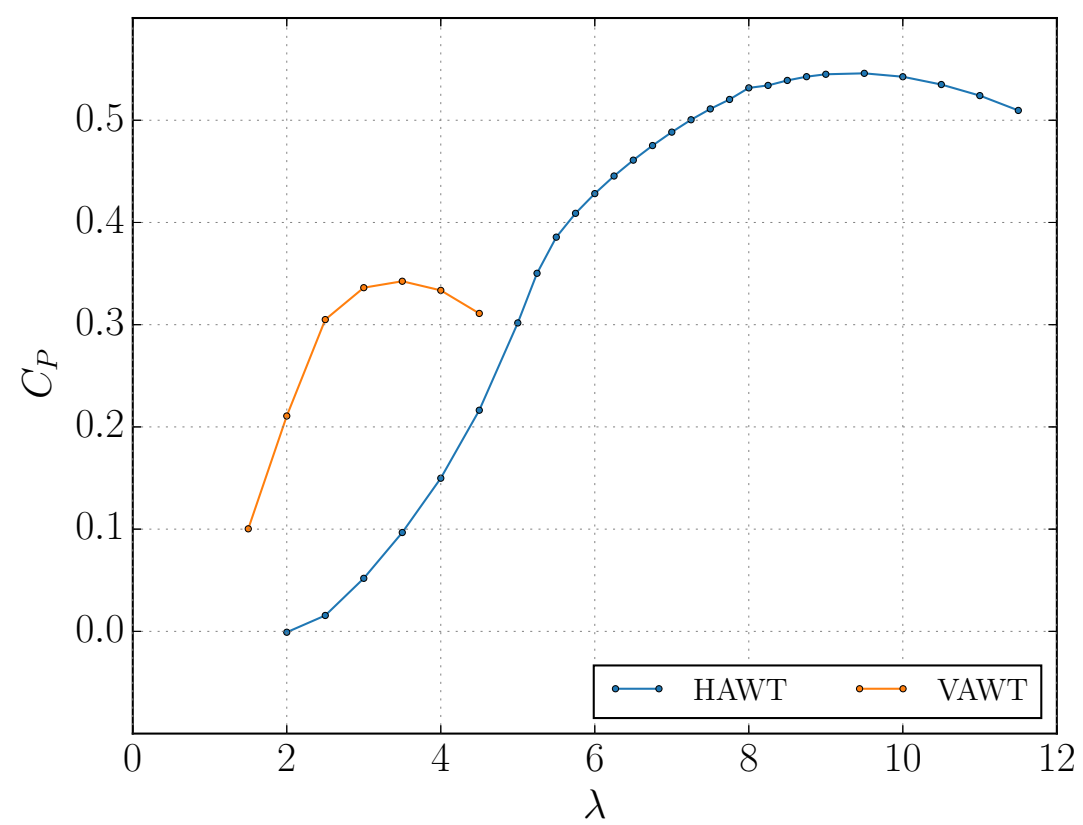

Figure 10: $C_{P}$ as funcion of $\lambda$ for both a VAWT and a HAWT in full scale.
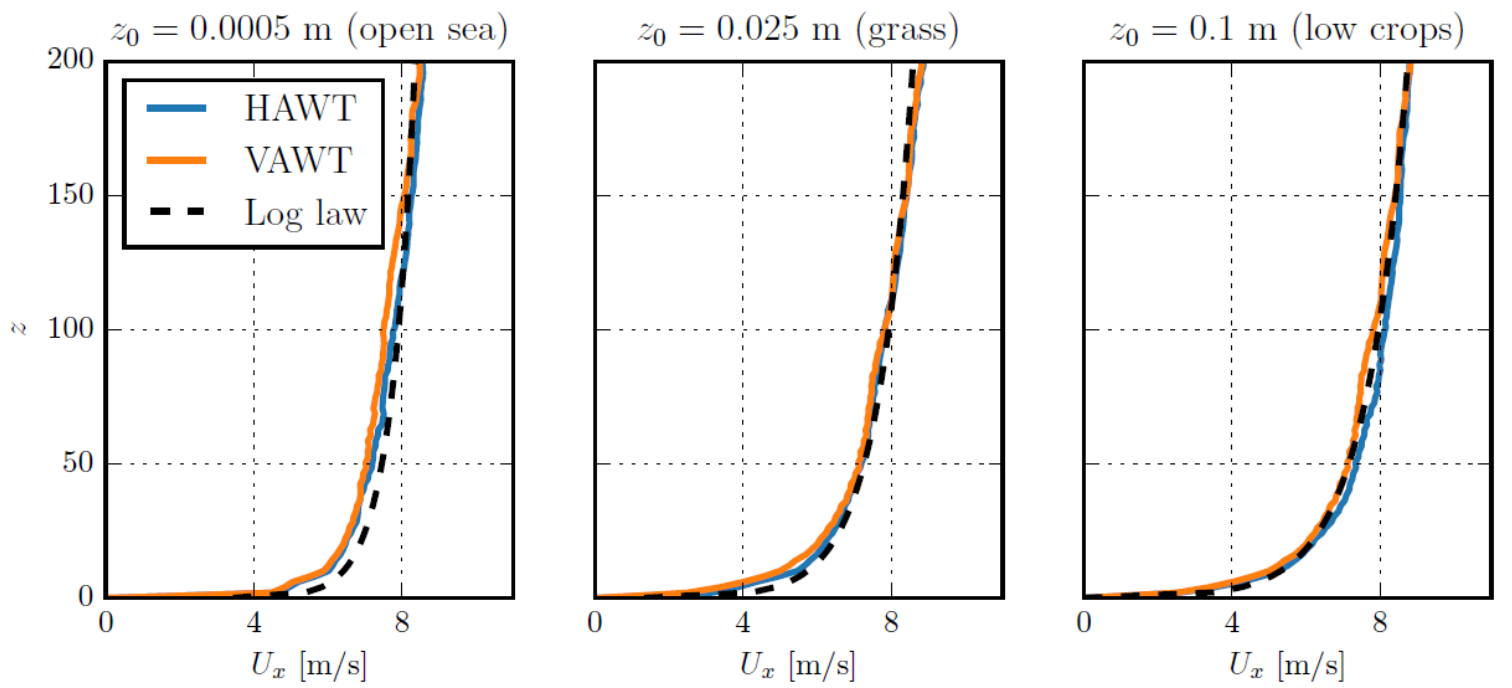

Figure 11: Vertical profile of the mean streamwise velocity for the inflow conditions. 
$U_{x} / V_{\infty}$

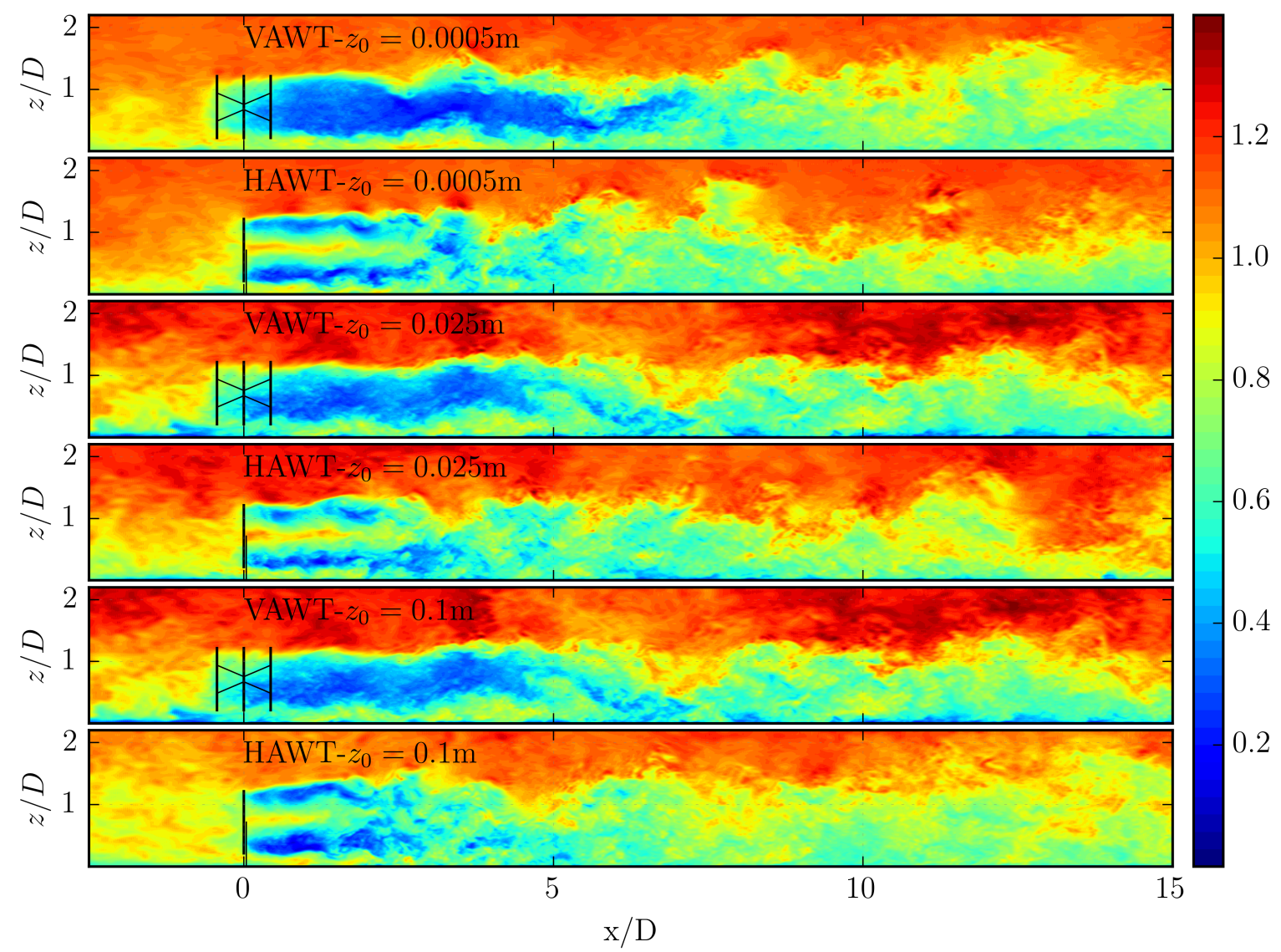

Figure 12: Normalized instantaneous streamwise velocity in the vertical plane at the centre of the turbine for different terrains. 


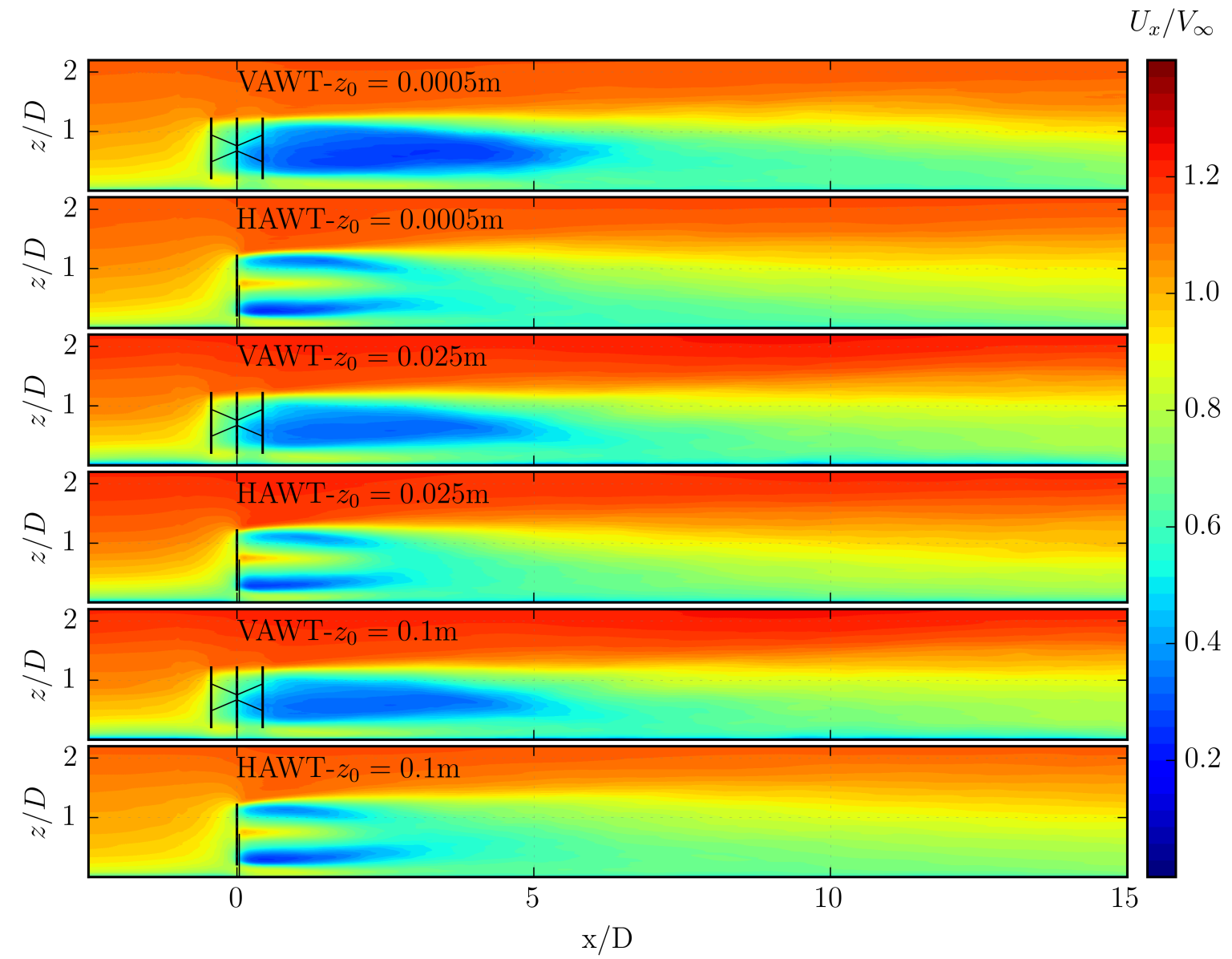

Figure 13: Normalized mean streamwise velocity in the vertical plane at the centre of the turbine for different terrains. 


\section{$U_{x} / V_{\infty}$}
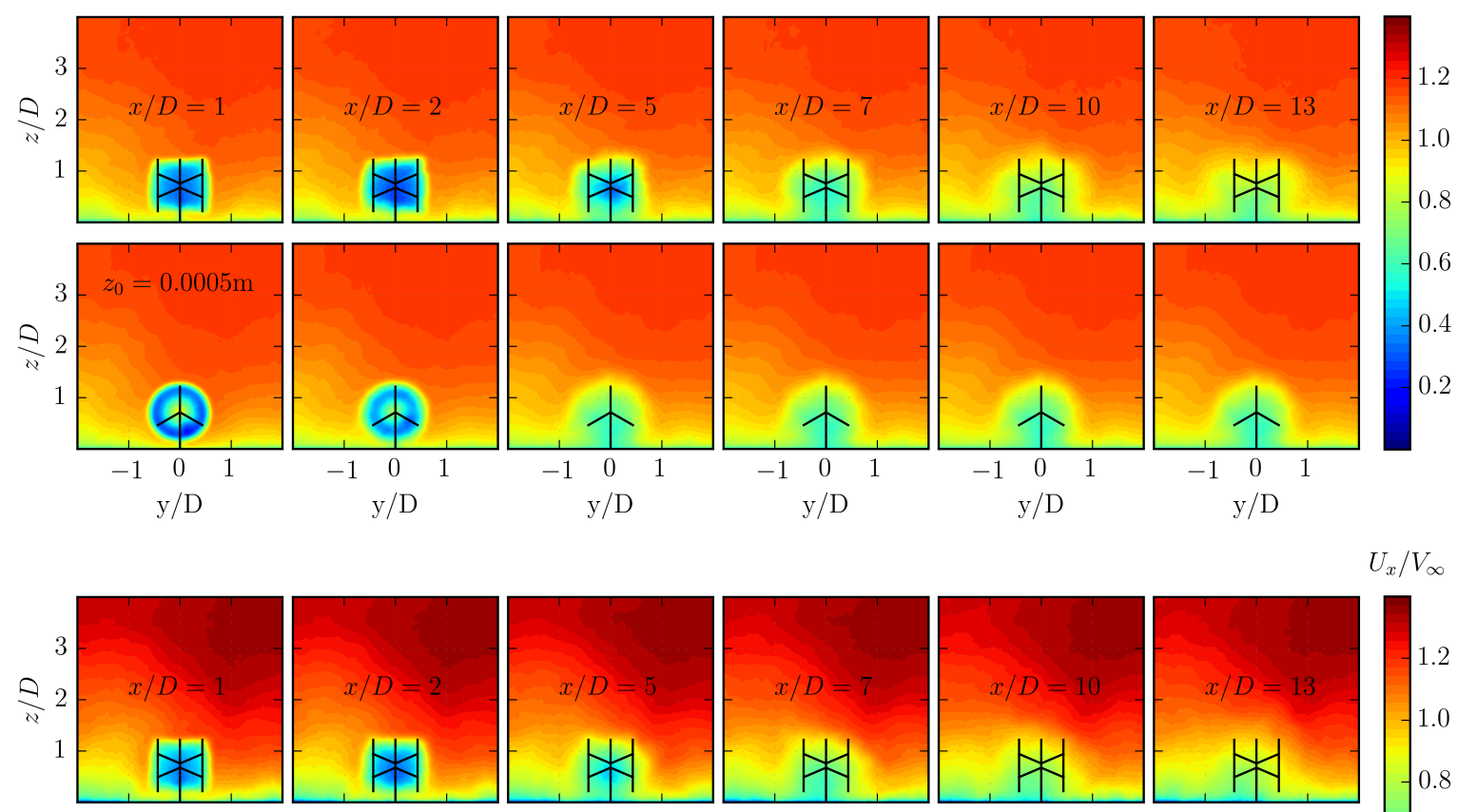

$U_{x} / V_{\infty}$
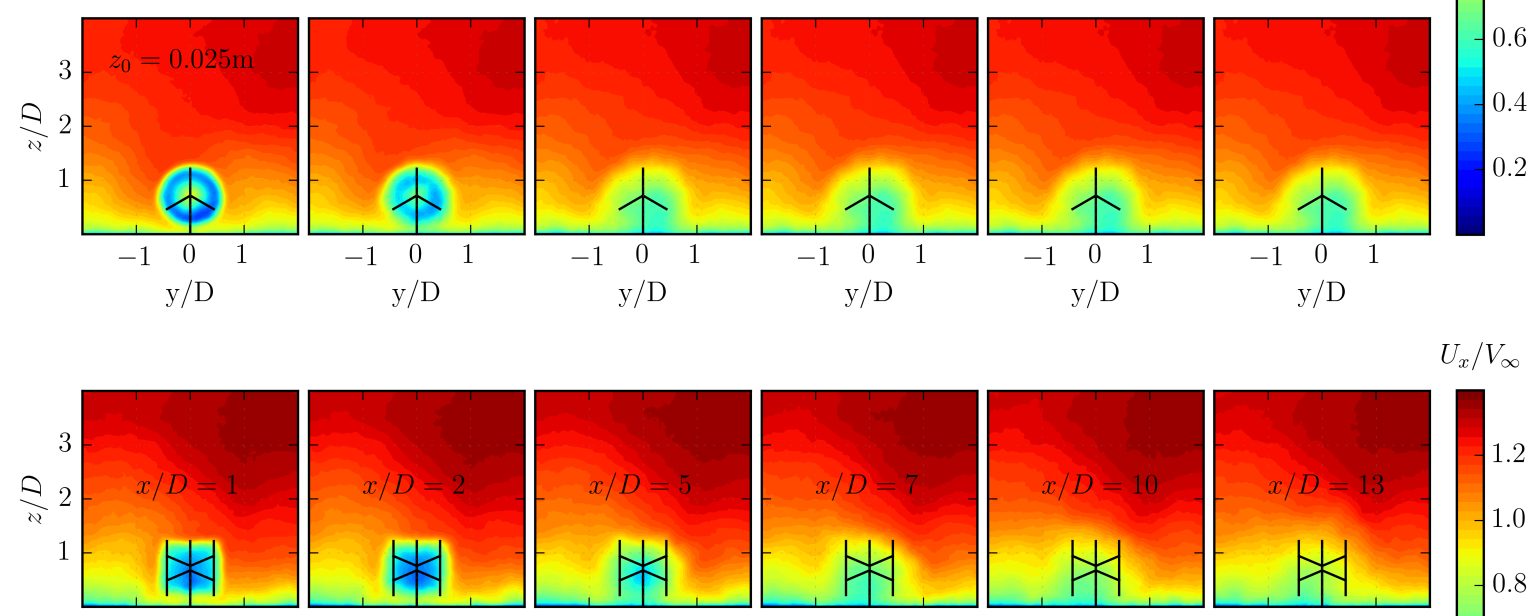

$U_{x} / V_{\infty}$
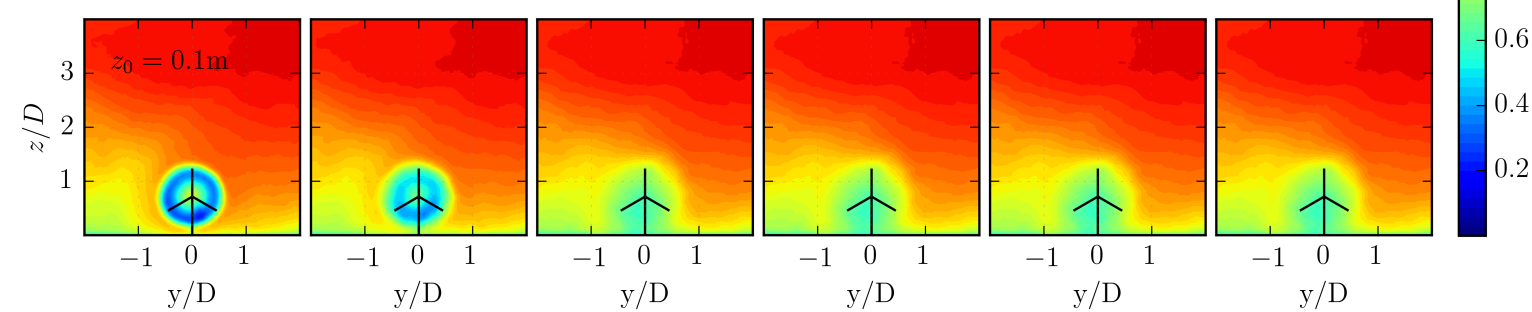

Figure 14: Normalized streamwise velocity at different representative sections perpendicular to the flow for different terrains with $z_{0}=0.0005 \mathrm{~m}$ (top), $0.025 \mathrm{~m}$ (center) and $0.1 \mathrm{~m}$ (bottom). 

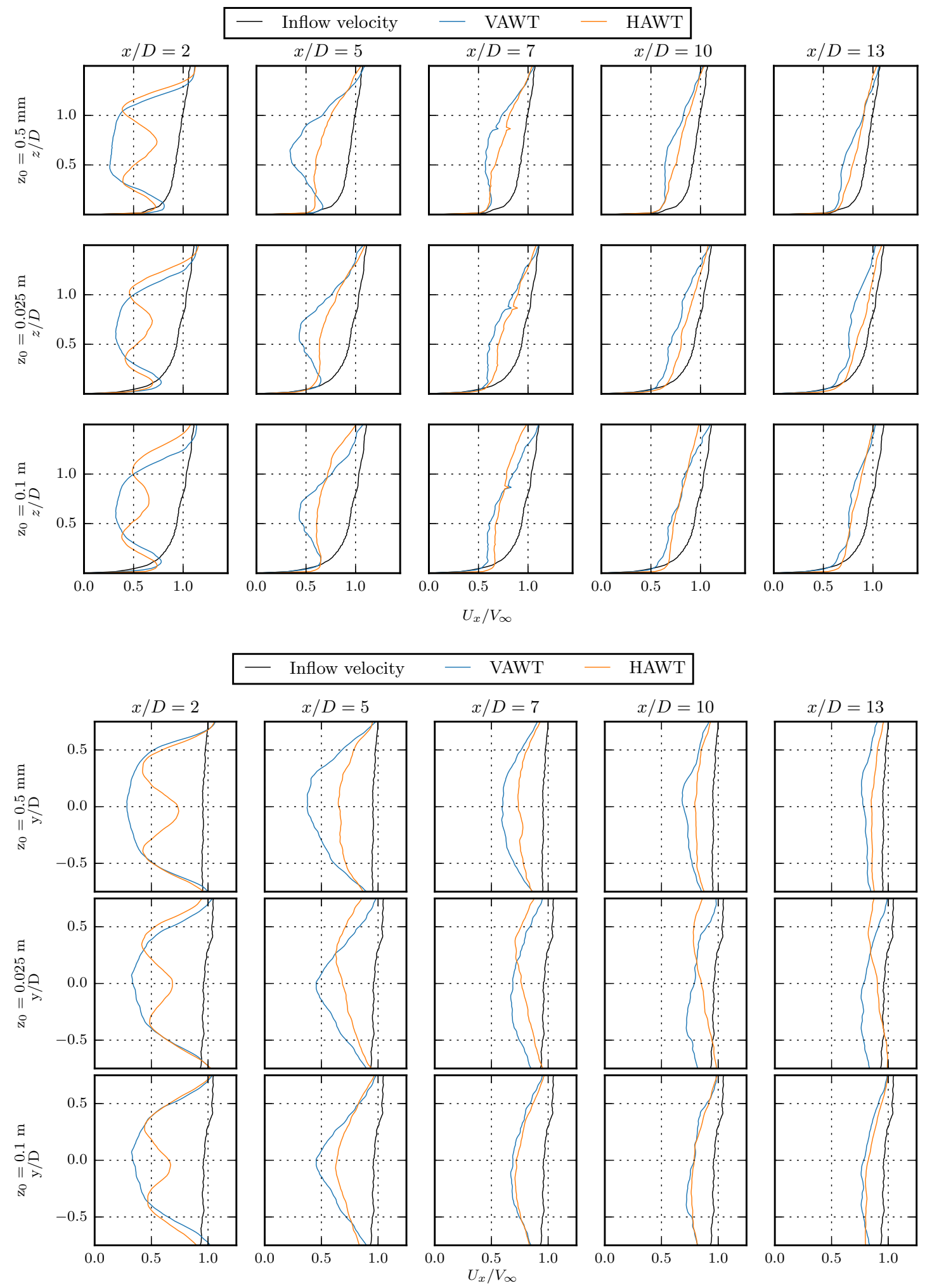

Figure 15: Vertical (up) and horizontal (down) profiles of the normalized velocity of the spanwise profiles of the normalized mean streamwise velocity at different representative downstream sections. 